\title{
A colloquio con Enrico Loccioni ${ }^{1}$
}

\section{di Claudio Baccarani e Gian Luca Gregori}

Buon pomeriggio a tutti, onorati di essere qui con Voi ed onorati di intervistare Enrico Loccioni, con il quale il sistema Universitario collabora sinergicamente da oltre 40 anni.

Perché un intervento del genere in questo convegno? Proprio nellaccezione allargata di Cultura, di Heritage, soprattutto in termini di tutela e di valorizzazione del territorio. Ciò nella consapevolezza che svolgere attività di impresa significa creare valore per il territorio e per la Comunità.

Una frase che ci ha colpito molto di Enrico è la seguente: "non chiamatela azienda, la mia è una bella avventura", costruita insieme alla moglie Graziella, colonna fondamentale nello sviluppo del percorso imprenditoriale, purtroppo recentemente scomparsa e considerata una "mamma da tutti i 400 «ragazzi» del gruppo".

Potremmo in un titolo sintetizzare cosi la traiettoria evolutiva del Gruppo Loccioni: "dalle mucche, allelettricista, ai produttori più importanti nel mondo!"; questo ad evidenziare innanzitutto il ruolo fondamentale che ha avuto il mondo agricolo, nel quale Enrico cresce e sviluppa la sua prima "invenzione": un sistema per abbeverare gli animali delle stalle in "modo automatico", grazie alla grande innovazione che fu l'arrivo dell'elettricità e ad i primi rudimenti di elettrotecnica appresi a scuola e quindi evitando di portare manualmente pesi rilevanti. Questa soluzione colpi molto i contadini della zona, che andavano a vedere (potremmo dire a "spiare") il risultato dell'ingegno di un giovane ragazzo. Ma il "tema" che poi Enrico ha sviluppato in tutta la sua vita è stato sempre lo stesso, come nel caso delle mucche: "partire dai bisogni e trovare soluzioni", anzi, il motto prevalente è: "dove ci sono problemi, allora siamo pronti ad intervenire perché là ci sono opportunità"!

Prima di iniziare la mia attività ho avuto una breve esperienza come dipendente in una fabbrica e allora ho capito che non amavo il lavoro ripetitivo e che non volevo più farlo e non volevo costringere qualcun altro a farlo. Per questo ho deciso di non orientare la mia attività su una produzione in serie. Ma ho subito capito che una decisione di questo tipo implicava il coinvolgimento del territorio, della scuola e la collaborazione con le migliori imprese e i migliori artigiani degli altri settori. Ho ripetuto più volte che il gruppo Loccioni ha cercato sempre di lanciare sassi nell'acqua per scatenare onde. Esattamente come quando lanciamo un sasso nell'acqua e si genera unonda che si allarga ma allo stesso tempo sembra tornare indietro, così l'impresa dovrebbe, attraverso le sue azioni, creare un'onda di interesse e di coinvolgimento che si diffonde nel territorio e torna indietro attraverso competenze e know how da poter reinvestire in nuovi progetti.

1 Intervista realizzata durante la sessione plenaria del 10 luglio 2015 in occasione del XXVII Convegno Annuale di Sinergie "Heritage, management e impresa: quali sinergie?", Termoli 9-10 luglio 2015. 
sinergie Vol. 34, N. 100, 2016

Léettricista è stato il primo vero lavoro di Enrico, che inizia in modo artigianale a realizzare impianti elettrici per varie imprese della zona; quindi organizza l'attività in modo più industriale, crescendo in termini dimensionali; ma è la sua visione che consente di allargare la prospettiva, ponendo unattenzione crescente al tema della misurazione ed alla trasformazione dei dati in valore. Una sua considerazione poneva al centro il benessere delle persone, considerando l'evoluzione delle metropoli: "dobbiamo essere in grado di misurare il livello di inquinamento, di utilizzo dellacqua, di energia....", per migliorare la qualità della vita delle persone; ed il concetto di benessere si è poi spostato anche agli animali, tema oggi molto di attualità, soprattutto riguardo alle condizioni di vita negli allevamenti intensivi.

Il gruppo Loccioni è un'impresa familiare che offre soluzioni integrate per migliorare la qualità ed aumentare la sostenibilità dei prodotti e dei processi. Uno degli aspetti che curiamo maggiormente è la misurazione dei dati perché attraverso di essa si acquista la consapevolezza di ciò che si fa.

Non amo parlare né di fabbrica, né di industria, per il semplice fatto che non facciamo attività manifatturiera, non abbiamo linee di produzione ma sviluppiamo progetti e ogni progetto è unavventura a sé.

Il vero obiettivo della nostra impresa è quello di migliorare la qualità della vita delle persone in modo sostenibile. Fare impresa per creare valore e valori insomma.

Da qui, la conquista della fiducia dei più grandi produttori nel mondo, nel settore automotive, degli elettrodomestici, dellenergia, dei trasporti, dell'aerospaziale, dell'agroalimentare e di recente anche nella sanità, con lo sviluppo del primo robot al mondo per la preparazione automatica delle dosi dei farmaci per le terapie oncologiche, annullando il rischio di tossicità per gli operatori della farmacia e la possibilità di errore nella preparazione degli stessi. Nella ricerca dei clienti, la strategia è: "tra i player più importanti del mondo"! In ogni settore si cerca la partnership con i leader di mercato dai quali raccogliere sfide innovative e stabilire relazioni di lungo periodo.

Come una sartoria dove ogni cliente richiede soluzioni in base alle proprie misure ed esigenze noi creiamo per le nostre cinque aree di business - energy, environment, industry, humancare e train and transport- reti di ricercatori che si scambiano informazioni per migliorare la qualità dei prodotti. Per esempio l'energia prodotta da fonti rinnovabili è intermittente e discontinua: noi abbiamo lavorato su sistemi di accumulo dellenergia integrando batterie di diverse tecnologie e taglie. In tal modo la produzione di energia verde diventa davvero più efficace e sostenibile.

L'accoglienza è un fattore determinante nella strategia del gruppo, non "artificioso", cioè costruito, ma diremmo "spontaneo". Non a caso è continuo il processo di conoscenza locale e globale basti pensare alla sede, nella Valle Esina, ad Angeli di Rosora. Così più volte, scherzando ma non troppo, $i$ collaboratori del Gruppo quando si presentano dicono che hanno sede a Los Angeles di Rosora, anche oggi che il Gruppo ha tre sedi, negli Stati Uniti, in Germania ed in Cina. Il Gruppo Loccioni si trova in aperta campagna. 
Nella sua sede circolano vari animali in libertà, tra cui gru coronate e pavoni impegnati spesso a guardarsi negli specchi appositamente collocati per soddisfare la loro vanità (in realtà il pavone allo specchio fa la ruota perché in sfida con il "rivale" di fronte a lui).

La saggezza contadina ed $i$ valori che ne derivano sono alla base di questo legame con il territorio come fattore competitivo. Cosi il padre di Enrico, che ne è espressione tipica, suole ripetere quando vede "persone troppo agitate": "mica devi fare tutto oggi, lasciati da fare qualcosa anche domani!".

Io credo di aver imparato tanto dalla tradizione e dalla cultura contadina: il senso di fiducia - gli accordi presi con una stretta di mano - di comunità, del sacrificio. Insomma lo sguardo alla tradizione per me è sempre stato fondamentale, però nello stesso tempo ho sempre pensato che la tradizione doveva darci degli spunti per creare qualcosa di nuovo, per innovare quindi e non per rimanere solo legati al passato. Uno studente che ci ha fatto visita durante uno dei laboratori aperti che organizziamo da tempo ha coniato il termine Tradinnovazione che io continuo ad usare perché ben riassume il mio sentire, il valore culturale di un territorio e della sua storia in una visione aperta al futuro. Credo che in un'impresa debbano sempre esserci energia, entusiasmo, passione e senso di responsabilità e che al suo interno sia necessario coltivare l'immaginazione che ci aiuta nella costruzione del futuro.

E questo legame con il territorio trova tangibile riscontro in due progetti: la Leaf Community e il Land of Value. Con la Leaf Community, è stato realizzato un progetto di riqualificazione energetica degli edifici industriali e di tutta l'area del "campus" Loccioni, inclusa la costruzione di una "casa intelligente", autonoma e per nulla impattante sull'ambiente, riprendendo il concetto della "casa contadina", autonoma nella sua gestione. In collaborazione con grandi gruppi internazionali, si è realizzata una comunità ecosostenibile e la prima micro-grid energetica in Italia, dimostrando che è possibile rendere operative progettualità di questo tipo.

Il Land of Value rappresenta un "metodo di valorizzazione" del territorio, ma anche un approccio distintivo che è alla base della costruzione di un' esperienza, che sembrerebbe cosi distante dalle soluzioni high tech proposte dall'impresa. Eppure funziona, con risultati sorprendenti! L'idea è semplice ed è quella di trasformare le tipicità del territorio in un fattore fortemente competitivo. Cosi l'accoglienza dei manager internazionali avviene ricorrendo ad una rete di agriturismi, dove l'occasione è quella di soggiornare e di usufruire dei prodotti eno-gastronomici locali. In alcuni casi i manager ritornano in vacanza con le famiglie in queste originali strutture ricettive. Cosi, le colazioni di lavoro non sono affidate ai tradizionali catering, ma agli artigiani locali agro-alimentari che illustrano i propri prodotti, rilevandone le principali caratteristiche. Anche in questo caso, si riscontrano risultati molto positivi, in termini di "qualità sorprendente delle relazioni".

Fare rete con il territorio, con le sue istituzioni, con la sua comunità culturale ed economica è fondamentale per noi. Tra vivere il territorio e semplicemente "alloggiarlo" noi abbiamo deciso di viverlo. L'impresa vive e prospera se vitale e prospero è il territorio che abita ed è sua responsabilità e
Claudio Baccarani
Gian Luca Gregori

A colloquio con Enrico Loccioni 
sinergie Vol. 34, N. 100, 2016

interesse fare in modo che lo sia. Nel 1990 la nostra impresa si è allagata a causa dell'esondazione del fiume Esino e siamo riusciti a riprendere il lavoro nel giro di una settimana solo grazie al lavoro di tutti i nostri collaboratori e delle loro famiglie. In seguito abbiamo deciso di prenderci cura dei $2 \mathrm{~km}$ del fiume tra i ponti di Scisciano e Apiro attraverso il ripristino del corso originario del fiume, il rinforzo degli argini e l'installazione dei sistemi per il monitoraggio sia del fiume che del ponte, abbiamo finanziato il rimboschimento dell'area e la costruzione di una pista ciclabile. Questo investimento è per noi un investimento sul futuro che fa riscoprire alle persone il valore della tradizione riportando un elemento naturale, come il fiume, ad essere una risorsa e non una minaccia. Ecco perché amiamo dire che abbiamo investito su $2 \mathrm{~km}$ di futuro. Come dice il protagonista dello spot che abbiamo girato per raccontare questo progetto "da grande voglio coltivare bellezza in $2 \mathrm{~km}$ di futuro.

\section{Il designer giapponese Isao Hosoe vi ha definito una Play Factory, in che senso?}

All'inizio questa definizione ci ha piuttosto sorpreso ma poi riflettendo abbiamo capito che essa riesce a racchiudere aspetti della nostra impresa che sfuggivano ad altre descrizioni: per esempio il fatto che non abbia una struttura gerarchizzata, non esistono infatti per noi dipendenti ma solo collaboratori che si divertono perché lavorano con passione e condividono le proprie conoscenze realizzandosi sia professionalmente che umanamente. Soprattutto i giovani hanno un ruolo determinante. I giovani sono curiosi e hanno voglia di approfondire e sentono profondamente la voglia di cambiamento. La nostra attività ha bisogno di persone aperte e non pre-impostate: è per questo che scegliamo sempre giovani senza esperienza e l'età media dei nostri collaboratori (per metà laureati) è di 32 anni. Consideri che un tempo i giovani terminavano la loro carriera universitaria e poi venivano chiamati in azienda a lavorare. Oggi quelli che contribuiscono allo sviluppo del nuovo sono quei ragazzi che comunque parallelamente allo studio sono riusciti con curiosità ad avvicinarsi al mondo che li ospiterà da lavoratori della conoscenza.

Nonostante le problematiche e le difficoltà che si possono incontrare, è sempre necessario tenere presente che se si sogna un progetto lo si può realizzare. Ed è questo l'augurio che voglio fare a tutti i giovani.

\section{Academic or professional position and contacts}

\section{Claudio Baccarani}

Full Professor of Management

University of Verona - Italy

ISSN 0393-5108 Gianluca Gregori

DOI 10.7433/s100.2016.08 Full Professor of Management

pp. 127-130 Politecnica delle Marche University - Italy

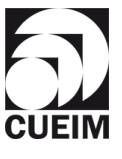

e-mail: g.gregori@univpm.it

Enrico Loccioni

Loccioni Group President- Italy

e-mail: e.loccioni@loccioni.com 